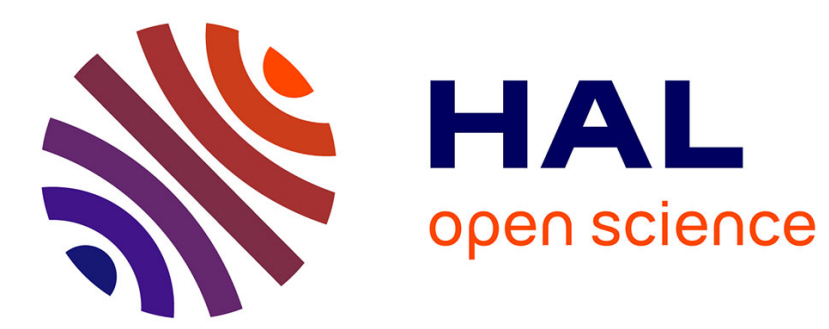

\title{
Zoom into dusty plasma instabilities
}

Hagop Tawidian, Thomas Lecas, Maxime Mikikian

\section{To cite this version:}

Hagop Tawidian, Thomas Lecas, Maxime Mikikian. Zoom into dusty plasma instabilities. IEEE Transactions on Plasma Science, 2013, 41, pp.754. hal-00744759

\section{HAL Id: hal-00744759 \\ https://hal.science/hal-00744759}

Submitted on 17 Dec 2012

HAL is a multi-disciplinary open access archive for the deposit and dissemination of scientific research documents, whether they are published or not. The documents may come from teaching and research institutions in France or abroad, or from public or private research centers.
L'archive ouverte pluridisciplinaire HAL, est destinée au dépôt et à la diffusion de documents scientifiques de niveau recherche, publiés ou non, émanant des établissements d'enseignement et de recherche français ou étrangers, des laboratoires publics ou privés. 


\title{
Zoom into dusty plasma instabilities
}

\author{
Hagop Tawidian, Thomas Lecas, and Maxime Mikikian
}

\begin{abstract}
In a krypton plasma, the growth of dust particles can strongly affect the plasma characteristics by inducing many types of instabilities. These unstable phenomena are studied by analyzing their frequency evolution as a function of time and by emphasizing the appearance of plasma spheroids. Different phases are evidenced thanks to a detailed analysis of the form and frequency of the discharge current. Concerning the plasma spheroids, interesting motions in the plasma bulk are evidenced.
\end{abstract}

Index Terms-Dusty plasma, complex plasma, dust particle growth, instabilities, Fourier analysis, plasma spheroids.

\section{INTRODUCTION}

$\mathbf{P}$ LASMAS are present in nature and also in many industrial processes. When favorable conditions are encountered, dust particles can be grown in these plasmas. The formation of dust particles has been intensively studied since their discovery in plasma processing reactors [1], [2] particularly in microelectronics. These particles can fall on wafers and affect semiconductor device performances. However, these nanometer size dust particles can be useful in some industrial applications like solar cells [3] or memories. More recently, the existence of dust particles in fusion reactors [4] like the future ITER, is the subject of a special attention. In nature, especially in the astrophysics field, dusty plasmas are found in comet tails and planetary atmospheres [5].

In laboratory, dust particles can be grown using reactive gases [6]-[13] or material sputtering [14]-[16], molecular precursors resulting from both methods launch a succession of chemical reactions leading to the growth of dust particles. In the present work, material sputtering is used to grow a high density of dust particles in a $\mathrm{Kr}$ plasma. During their growth, they acquire a negative charge by capturing electrons [17]-[19]. This loss of free electrons [20] can lead to a disturbance of the plasma equilibrium. Many types of unstable behaviors can be observed like the heartbeat instability [21], [22], dust particle growth instabilities (DPGI) [23], [24] or rotating plasma spheroids close to the electrodes [25], [26]. To study the evolution of the instabilities as a function of time, the amplitude of the discharge current fundamental harmonic is recorded and analyzed using different methods like the Fourier spectrogram, Fourier spectrum and peak detection. Several regimes are identified with different frequencies and signal forms. One of the objectives of this work is to find a way to correlate the instability characteristics and the time evolution of dust particle size and density. It can open new perspectives for dust particle growth monitoring with an easily

H. Tawidian, T. Lecas, and M. Mikikian are with the GREMI, Groupe de Recherches sur l'Energétique des Milieux Ionisés, UMR7344, CNRS/Université d'Orléans, 45067 Orléans Cedex 2, France e-mail: (hagop.tawidian@univ-orleans.fr and maxime.mikikian@univ-orleans.fr). implemented diagnostics. A good characterization of these instabilities has to be performed as a first step. In the present paper, this characterization is explored. Thanks to a highspeed camera, a new phenomenon has also been observed by recording the plasma glow. Plasma spheroids characterized by a slightly enhanced luminosity appear stochastically in the plasma bulk. These spheroids can have up and down motions and sudden changes in their directions.

\section{EXPERIMENTAL SETUP}

Experiments are performed in the PKE-Nefedov reactor [15], [27] where the plasma is created by a capacitivelycoupled radio-frequency (13.56 MHz in push-pull mode) discharge in krypton. The two electrodes are separated by $3 \mathrm{~cm}$ and have a diameter of $4 \mathrm{~cm}$. The injected $\mathrm{rf}$ power is around $3 \mathrm{~W}$ for a pressure of 1.6 mbar. Dust particles are grown by sputtering previously injected micrometer size particles made of polymer (melamine formaldehyde) and lying on the electrodes. After each experiment, the grown dust particles fall down on the electrodes, and this additional material is also sputtered and involved in the growth process. In previous experiments with argon, the dust cloud was observed by laser light scattering using a thin laser sheet injected through the reactor by a laser diode at $685 \mathrm{~nm}$. However, when using $\mathrm{Kr}$, the dust cloud is not observed except in the afterglow phase, meaning that probably the dust particle size does not exceed $100 \mathrm{~nm}$. Indeed, in $\mathrm{Kr}$ the final size of the grown dust particles appears much smaller than in Ar while their number density is much higher. The $\mathrm{Kr}$ mass being twice higher than the Ar one, it can change the sputtering efficiency of the deposited material and also the dust particle erosion in the plasma volume. It can contribute to explain the higher dust particle density and the smaller final size leading to new behaviors not observed in Ar. To monitor dust particle growth, the amplitude of the discharge current fundamental harmonic is recorded. The plasma glow is observed thanks to a high-speed camera (Photron Fastcam SA5) at 16000 frames per second.

\section{DUST PARTICLE GROWTH INSTABILITIES AND THEIR DIFFERENT PHASES}

To obtain a sufficiently high dust density, the reactor was pumped down to a very low base pressure around $10^{-6}$ mbar before each experiment. Once the plasma is switched on, the sputtering process begins and after a few tens of seconds, the instability beginning is observed. The loss of free electrons on the dust particle surface is observed on the amplitude of the discharge current that is decreasing during dust particle formation and growth (Fig. 1). Sudden amplitude drops in the electrical signal are noticed when using $\mathrm{Kr}$. These drops last for a few seconds and are evidenced just after the plasma 


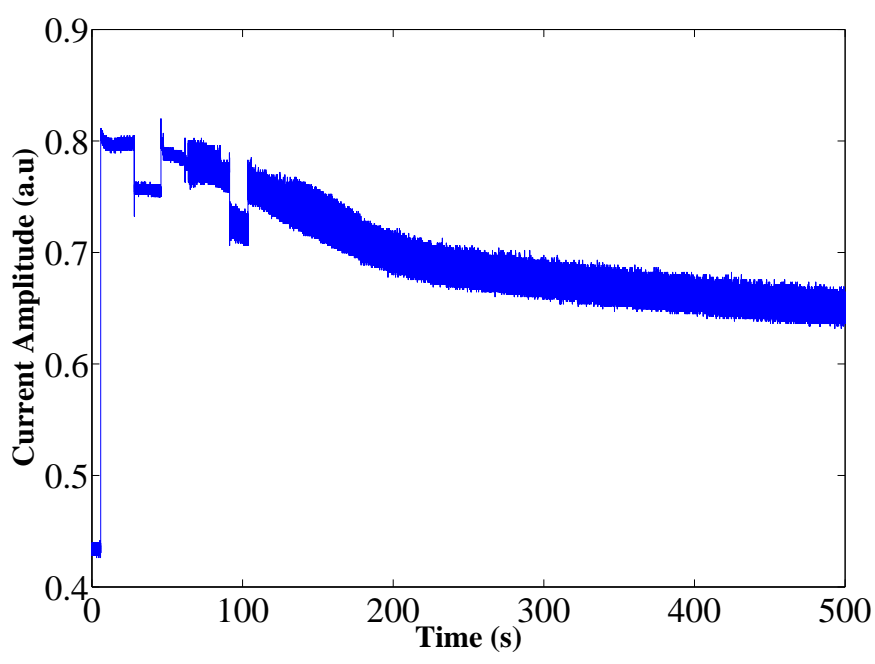

Fig. 1. Time evolution of the amplitude of the discharge current during dust particle growth (dc component). DPGI begin at $62 \mathrm{~s}$. Sudden drops are observed at $28 \mathrm{~s}$ and $91 \mathrm{~s}$ and could be related to the high dust particle density.

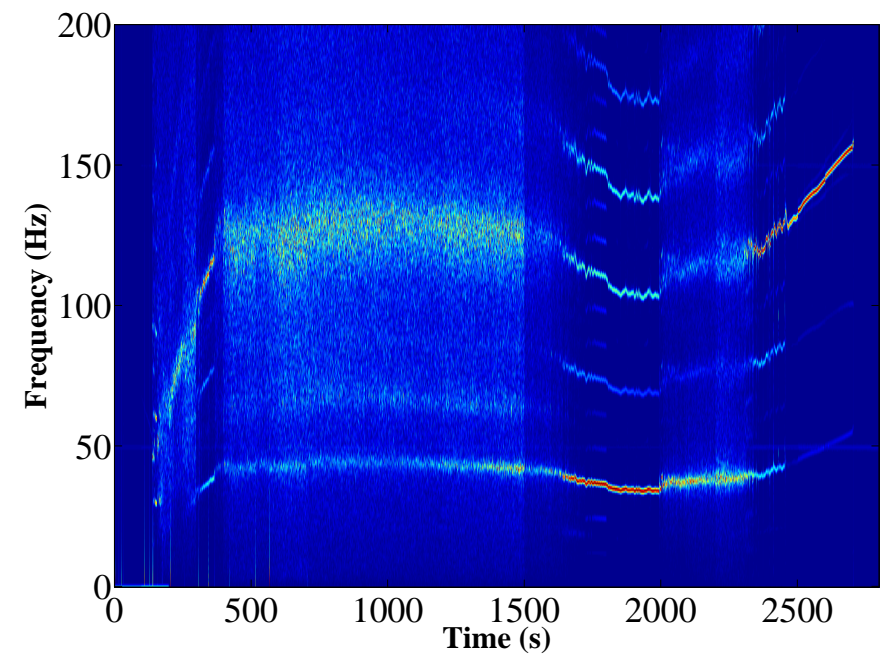

Fig. 2. Fourier spectrogram of the electrical measurements showing the frequency evolution as a function of time.

ignition and during the first part of the instabilities. Later in the growth process, the existence of these drops is no more evidenced. Their origin is currently under investigation and could be related to the huge density of grown dust particles.

To study more precisely the evolution of the instabilities as a function of time, the oscillating part of the electrical measurements is recorded to have a better oscilloscope vertical resolution. This measurement is performed during a new experiment, different than the one presented in Fig. 1. The Fourier spectrogram of the signal is calculated to distinguish different phases in DPGI (Fig. 2). To highlight the importance of the different frequencies, the Fourier spectrogram intensity has been normalized on each $100 \mathrm{~s}$ range. The instability begins around $140 \mathrm{~s}$ after the plasma ignition and consists of a succession of regular and chaotic phases. In a global way, a scenario tends to repeat. Several ordered phases are detected just after the instability beginning and are followed

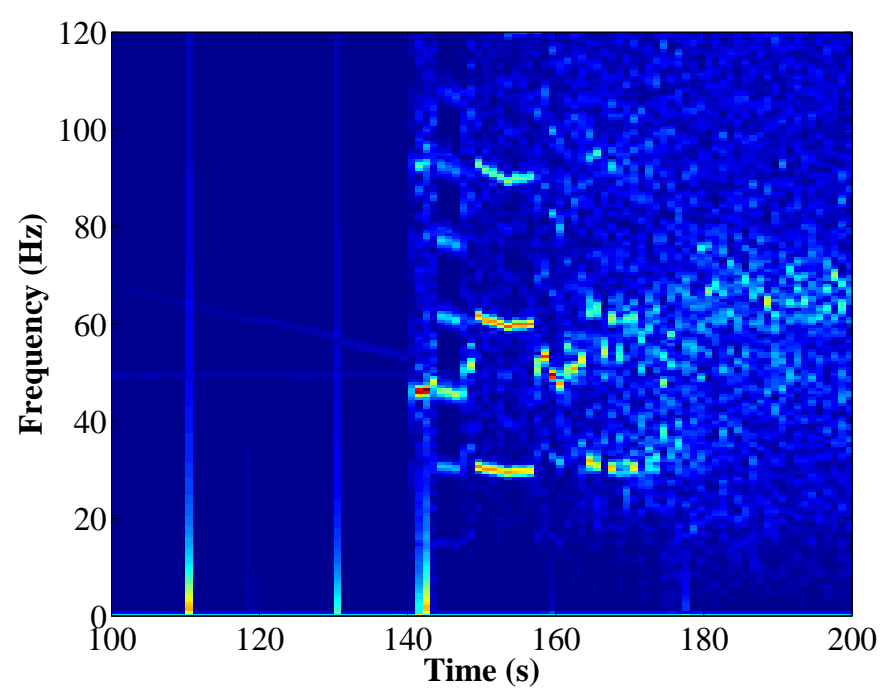

Fig. 3. Zoom of Fig. 2 on the first part of the instability. The instability begins at $140 \mathrm{~s}$ and is characterized by 4 successive ordered phases before entering in a chaotic regime.

by a long chaotic phase (between $162 \mathrm{~s}$ and $1400 \mathrm{~s}$ ) briefly interrupted by a short ordered phase (between $300 \mathrm{~s}$ and $370 \mathrm{~s}$ ). Then, a regular phase occurs with a decreasing frequency, and finally, the instability stops after another short chaotic phase that becomes more and more regular.

In Fig. 3, a zoom of the Fourier spectrogram is made to study more specifically the instability beginning, the vertical lines around (110 s and $130 \mathrm{~s}$ ) correspond to electrical drops similar to the ones observed in Fig. 1. The ordered phases are well observed between $140 \mathrm{~s}$ and $162 \mathrm{~s}$. Four phases can be clearly evidenced. The first phase from $140 \mathrm{~s}$ to $142 \mathrm{~s}$ is followed by a second one until $148 \mathrm{~s}$. Then, a well-defined third phase takes place until $157 \mathrm{~s}$ and finally a fourth one (less clear) exists until $162 \mathrm{~s}$ where the system enters in the chaotic regime.

In order to better characterize these phases, the corresponding electrical signals and their Fourier spectra are presented in Fig. 4. All the phases show well-defined patterns containing a varying number of peaks. For the first phase (Fig. 4(a)), the main pattern is composed of two big peaks followed by a smaller one while for the second and third phases, respectively four (Fig. 4(b)) and two peaks (Fig. 4(c)) of different amplitudes form the main pattern. The fourth phase (Fig. 4(d)) appears roughly similar to the first one with differences in the relative amplitude of the peaks. From this first insight, the transition between each phase is characterized by the appearance or disappearance of peaks. The frequency evolution is also quite interesting. In order to better understand and complete Fig. 3, the Fourier spectrum of each phase is presented in Figs. 4(e)-(h). It appears that the same typical frequencies or their harmonics are globally encountered in each phase. Harmonics are easily obtained due to the nonsinusoidal shape of the signals that have similarities with sawtooth signals. The smallest detected frequency is about $15-16 \mathrm{~Hz}$. This frequency is clearly detected in Fig. 4(f), is very small in the cases corresponding to Figs. 4(e) and (h) and 

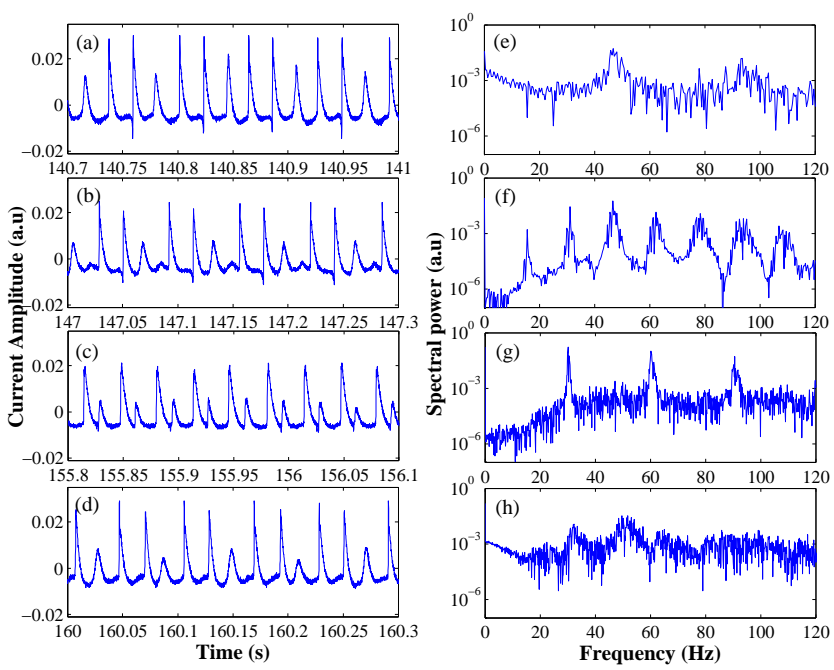

Fig. 4. Electrical signals and their corresponding Fourier spectra for the first 4 ordered phases of the instability.

cannot be detected in Fig. 4(g). Whereas in Figs. 4(e) and (h) presenting the whole frequency range this frequency cannot be observed, its presence has been confirmed by filtering and zooming the corresponding data around $15 \mathrm{~Hz}$. In the phases where this frequency appears, it corresponds to the main pattern frequency that is slightly similar in phases 1,2 and 4 . For phase 3 , the main pattern frequency is about 30 $\mathrm{Hz}$, twice the value of the other phases. This value of 30 $\mathrm{Hz}$ is present in all the phases, as a main frequency or as an harmonic. Another interesting value is the frequency at $45 \mathrm{~Hz}$ that is clearly present in phases 1,2 and 4 . In the Fourier spectra, a peak is centered at this value with a width of about $4 \mathrm{~Hz}$. It appears that $45 \mathrm{~Hz}$ is roughly the frequency corresponding to the peak separation within the main patterns. For phase 1, three peaks at a repetition rate of $45 \mathrm{~Hz}$ constitute the main pattern that repeats at $15 \mathrm{~Hz}$. For phase 2, the main pattern frequency is similar but with four peaks: The first two peaks appear at $45 \mathrm{~Hz}$ but the frequency for the two other peaks is continuously increasing with values multiple of 15 $\mathrm{Hz}$. The fact that the system oscillates at very well-defined frequencies that are multiple from each others, underlines that the dynamics behind these instabilities is relatively complex. For example, the sum of two signals at $30 \mathrm{~Hz}$ and $60 \mathrm{~Hz}$ can roughly reproduce the signal of phase 3 (Figs. 4(c) and (g)) but a linear combination of simple signals cannot reproduce the other phases in a satisfactory way. Additional experiments and analyses will be performed in order to better understand the frequency dependence of the signals and the occurrence and evolution of defined frequencies.

During the instabilities, the electrical signal can also be analyzed through the variations of its oscillation amplitude. The same time series data used to obtain Fig. 2 and to derive the results in Figs. 3 and 4 is also used to analyze the electrical signal data. To analyze this signal, the peak maxima and minima are measured. One part of the instability that appears particularly interesting corresponds to the region between 2360 $\mathrm{s}$ and $2460 \mathrm{~s}$. The peak detection of this region is shown in Fig. 5 where the maxima and the minima are represented.

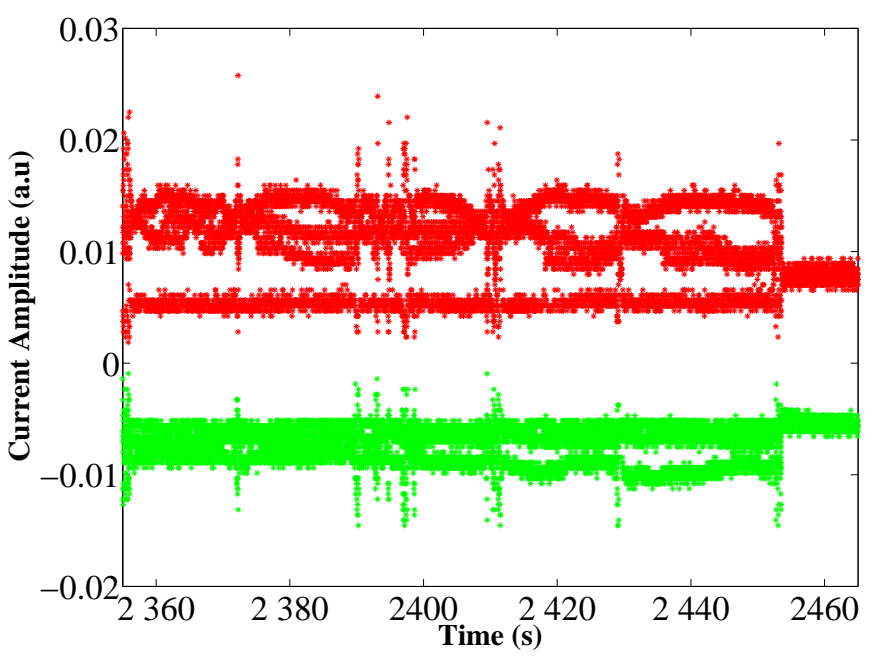

Fig. 5. Peak detection on the electrical measurements showing the evolution of the peak amplitudes. The maxima $(>0)$ and minima $(<0)$ are represented.

On the time series, this region is characterized by a 3 peak pattern and occurs just before the last ordered phase at the end of the instabilities. In Fig. 5, the maxima evolution shows a very particular behavior. It is especially clear at $2420 \mathrm{~s}$ where the amplitude evolution of the different peaks follows an eyeshape form. An increase of the amplitude of the main peak corresponds to the decrease of the amplitude of the second peak. Then, when the main peak reaches its maximum amplitude, it starts to decrease while the second peak amplitude behaves in the opposite way. When the amplitude of these two peaks becomes comparable, a short "chaotic" regime occurs that conclude the eye-shape oscillation. This phenomenon repeats several times during the instability. It is noteworthy to mention that the third peak keeps a constant amplitude during the whole duration of this phenomenon. Concerning the amplitude of the peak minima, its variation is not so clear and its analysis will be further examined. From these new analyses based on the amplitude variations, it appears that the resulting measured signal can be a complex combination of signals and plasma behaviors. They can evolve simultaneously or interact with each other, this interaction can be the consequence of the short chaotic regime occurring at the end of the eye-shape form when the two maxima have close amplitudes.

\section{Plasma Glow Behavior}

In the previous section, an analysis has been performed on the electrical measurements during the instabilities. In order to better understand the plasma evolution during DPGI, the plasma glow has also been analyzed by high-speed imaging. An interesting phenomenon consisting of the appearance of plasma spheroids in the plasma bulk is observed. These spheroids are well-defined regions of about a few $\mathrm{mm}$ with a slightly enhanced luminosity. In a previous paper [26] where experiments were performed in Ar, these spheroids where shown to appear and disappear near the electrodes. Their behavior was either "chaotic", or regular with clear rotations along the circumference of the electrodes [25], [26]. In this paper where $\mathrm{Kr}$ is used, these plasma spheroids are also 
evidenced in the plasma bulk as shown in Fig. 6 where 10 successive images are extracted from a video recorded at 16000 images per second. False colors are used to enhance the luminosity contrast in different regions of the plasma. The top and bottom regions of each frame correspond to the electrodes. As for Ar, many plasma spheroids appear in the vicinity of the electrodes. In the plasma bulk, these plasma spheroids have a comet-like shape as clearly seen for example in the first image of Fig. 6. The spheroid in the plasma center is heading toward the lower electrode where another spheroid seems to attract it as observed in the first 2 frames. Then, a sudden change in the spheroid direction occurs: Another spheroid emerges from the top presheath region and moves toward the plasma center. This new spheroid seems to impact the central spheroid as confirmed by looking at its comet-like shape: The comet head is now on the spheroid top (image 4 in Fig. 6). The central spheroid is now moving toward the top electrode up to image 10. These up and down motions can repeat several times. The composition of these spheroids is still unknown, they can be composed of just plasma or of plasma containing dust particles. Similar behaviors have been observed in a $\mathrm{Kr}$ plasma without dust particles [28] but with the insertion of an object in the plasma. As dust particles, this object can strongly disturb the free electron density and it can be suggested that similar instabilities with plasma spheroids can also be obtained. The origin of these plasma spheroids and particularly of their motion is currently under investigation. It raises interesting questions about the type of interaction between these regions of enhanced emission.

The correlation between the electrical measurements presented in section III and the plasma spheroids is difficult for several reasons. The electrical measurements are a global measurement, and thus are representative of the changes occurring in the whole plasma. Frames in Fig. 6 show a part of the plasma and do not give information on the possible presence of other spheroids in the plasma regions outside the camera field of view. Thus, a direct correlation would need to perfectly identify all spheroids appearing in the discharge. Concerning the spheroid motions, the correlation is even more difficult as a spheroid moving in the plasma does not necessarily change the discharge current. Indeed, if the "charge" transported by the spheroid is just displaced in the plasma it does not change the global current, except of course if the spheroid size and brightness are changing.

\section{CONClusion}

In this paper, instabilities triggered by the presence of dust particles are studied in a $\mathrm{Kr}$ plasma. These instabilities appear when a high density of grown dust particles is achieved. These instabilities are characterized by electrical measurements and high-speed imaging. The amplitude of the discharge current decreases due to the loss of free electrons on the dust particle surface. Some electrical drops are also evidenced and are currently under investigation. A Fourier analysis of the electrical measurements shows the existence of different phases during DPGI. The first 4 ordered phases are studied more precisely using the Fourier spectrum and by observing the signal shape.

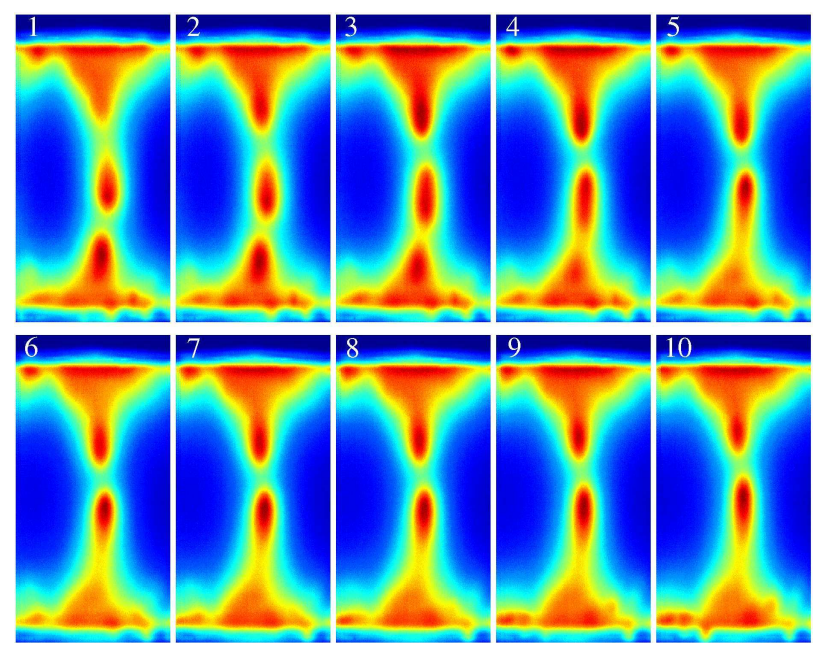

Fig. 6. Up and down motions of plasma spheroids in between the electrodes. Interactions between plasma spheroids can be detected. Images are extracted from a video recorded at 16000 frames per second.

After these regular phases, the instability enters into a long chaotic regime followed by a regular phase with a decreasing frequency. The instability ends with a short chaotic phase that becomes more and more regular. In comparison with Ar results [23] some preliminary comparisons can be made. Similarities are found in the succession of the different phases. As in $\mathrm{Ar}$, in $\mathrm{Kr}$ we observed that DPGI start with several regular regimes followed by a long chaotic regime and finish by a more regular phase. Concerning the frequency values, in $\mathrm{Kr}$ the frequencies are slightly higher than in Ar. This is consistent with the fact that the dust particle size is smaller and the dust particle density is higher in $\mathrm{Kr}$. A deeper comparison is currently on-going. The instability amplitude is also analyzed by using peak detection. This method shows that during some time periods, the peak maxima and minima follow a welldefined pattern consisting of the appearance of an eye-shape form. These various analyses show that the measured electrical signal is a complex combination of plasma behaviors with different typical frequencies.

In order to better investigate the plasma evolution during DPGI, the plasma glow has been analyzed by a high-speed camera evidencing the appearance of plasma spheroids in the plasma bulk. These spheroids have a comet-like shape with a slightly enhanced luminosity and they can move. Sudden changes in their direction appear to be due to the appearance of other nearby spheroids. The origin of the spheroid motions and interactions is currently under investigation and can reveal very interesting phenomena related to interaction models.

\section{ACKNOWLEDGMENT}

The authors would like to thank B. Dumax for electronic support, J.-M. Bauchire and H. Rabat for providing the high-speed camera. The PKE-Nefedov chamber has been made available by the Max-Planck-Institute for Extraterrestrial Physics, Germany, under the funding of DLR/BMBF under Grant No. 50WM9852. This work was partly supported by the French National Research Agency (ANR), project INDIGO ${ }^{\circ}$ ANR-11-JS09-010-01. 


\section{REFERENCES}

[1] R. M. Roth, K. G. Spears, G. D. Stein, and G. Wong, "Spatial dependence of particle light scattering in an rf silane discharge," Appl. Phys. Lett., vol. 46, p. 253, 1985.

[2] G. S. Selwyn, J. Singh, and R. S. Bennett, "In situ laser diagnostic studies of plasma-generated particulate contamination," J. Vac. Sci. Technol. A, vol. 7, p. 2758, 1989.

[3] P. Roca i Cabarrocas, P. Gay, and A. Hadjadj, "Experimental evidence for nanoparticle deposition in continuous argon-silane plasmas: Effects of silicon nanoparticles on film properties," J. Vac. Sci. Technol. A, vol. 14 , p. 655,1996

[4] S. I. Krasheninnikov, A. Y. Pigarov, R. D. Smirnov, M. Rosenberg, Y. Tanaka, D. J. Benson, T. K. Soboleva, T. D. Rognlien, D. A. Mendis, B. D. Bray, D. L. Rudakov, J. H. Yu, W. P. West, A. L. Roquemore, C. H. Skinner, J. L. Terry, B. Lipschultz, A. Bader, R. S. Granetz, C. S Pitcher, N. Ohno, S. Takamura, S. Masuzaki, N. Ashikawa, M. Shiratani, M. Tokitani, R. Kumazawa, N. Asakura, T. Nakano, A. M. Litnovsky, R. Maqueda, and the LHD Experimental Group, "Recent progress in understanding the behavior of dust in fusion devices," Plasma Phys. Control. Fusion, vol. 50, p. 124054, 2008.

[5] E. Sciamma-O'Brien, N. Carrasco, C. Szopa, A. Buch, and G. Cernogora, "Titan's atmosphere: An optimal gas mixture for aerosol production?" Icarus, vol. 209, p. 704, 2010.

[6] J. Berndt, E. Kovačević, I. Stefanović, O. Stepanovic, S. H. Hong, L. Boufendi, and J. Winter, "Some aspects of reactive complex plasmas," Contrib. Plasma Phys., vol. 49, p. 107, 2009.

[7] K. De Bleecker, A. Bogaerts, and W. Goedheer, "Detailed modeling of hydrocarbon nanoparticle nucleation in acetylene discharges," Phys. Rev. $E$, vol. 73, p. 026405, 2006.

[8] V. Massereau-Guilbaud, J. Pereira, I. Géraud-Grenier, and A. Plain, "Influence of the power on the particles generated in a low pressure radio frequency nitrogen-rich methane discharge," J. Appl. Phys., vol. 105 , p. 033302, 2009.

[9] J. Berndt, E. Kovačević, I. Stefanović, and L. Boufendi, "Controlled dust formation in pulsed rf plasmas," J. Appl. Phys., vol. 106, p. 063309, 2009.

[10] M. Mikikian, M. Cavarroc, L. Couëdel, Y. Tessier, and L. Boufendi, "Dust particles in low-pressure plasmas: Formation and induced phenomena," Pure Appl. Chem., vol. 82, p. 1273, 2010.

[11] M. Hundt, P. Sadler, I. Levchenko, M. Wolter, H. Kersten, and K. Ostrikov, "Real-time monitoring of nucleation-growth cycle of carbon nanoparticles in acetylene plasmas," J. Appl. Phys., vol. 109, p. 123305, 2011.

[12] M. Calafat, P. Yuryev, A. Drenik, A. Slim, and R. Clergereaux, "Carbon nanoparticle/hydrogenated amorphous carbon composite thin films formed in ecr plasma," Plasma Process Polym., vol. 8, p. 401, 2011.

[13] I. Géraud-Grenier, R. Jaffiol, V. Massereau-Guilbaud, and A. Plain, "Photoluminescence of hydrogen amorphous carbon nitrile particles obtained in a $13.56 \mathrm{mhz}$ dusty plasma," Appl. Phys. Lett., vol. 99, p. 091503, 2011.

[14] D. Samsonov and J. Goree, "Instabilities in a dusty plasma with ion drag and ionization," Phys. Rev. E, vol. 59, p. 1047, 1999.

[15] M. Mikikian, L. Boufendi, A. Bouchoule, H. M. Thomas, G. E. Morfill, A. P. Nefedov, V. E. Fortov, and the PKE-Nefedov Team, "Formation and behavior of dust particle clouds in a radio-frequency discharge: results in the laboratory and under microgravity conditions," New J. Phys., vol. 5, p. $19,2003$.

[16] C. Arnas, A. Mouberi, K. Hassouni, A. Michau, G. Lombardi, X. Bonnin, F. Bénédic, and B. Pégourié, "Carbon dust formation in a cold plasma from cathode sputtering," J. Nucl. Mater., vol. 390-391, p. 140, 2009.

[17] A. Melzer, T. Trottenberg, and A. Piel, "Experimental determination of the charge on dust particles forming coulomb lattices," Phys. Lett. A, vol. 191, p. 301, 1994.

[18] B. Walch, M. Horanyi, and S. Robertson, "Measurement of the charging of individual dust grains in a plasma," IEEE Trans. Plasma Sci., vol. 22, p. $97,1994$.

[19] C. Arnas, M. Mikikian, and F. Doveil, "Micro-sphere levitation in a sheath of a low pressure continuous discharge," Phys. Scr, vol. T89, p. 163, 2001.

[20] H. Kersten, H. Deutsch, M. Otte, G. H. P. M. Swinkels, and G. M. W. Kroesen, "Micro-disperse particles as probes for plasma surface interaction," Thin Solids Films, vol. 377-378, p. 530, 2000.

[21] M. Mikikian, M. Cavarroc, L. Couëdel, Y. Tessier, and L. Boufendi, "Mixed-mode oscillations in complex plasma instabilities," Phys. Rev. Lett., vol. 100, p. 225005, 2008.
[22] M. Mikikian, L. Couëdel, M. Cavarroc, Y. Tessier, and L. Boufendi, "Threshold phenomena in a throbbing complex plasma," Phys. Rev. Lett., vol. 105, p. 075002, 2010.

[23] M. Mikikian, M. Cavarroc, L. Couëdel, and L. Boufendi, "Low frequency instabilities during dust particle growth in a radio-frequency plasma," Phys. Plasmas, vol. 13, p. 092103, 2006.

[24] M. Mikikian, L. Couëdel, M. Cavarroc, Y. Tessier, and L. Boufendi, "Plasma emission modifications and instabilities induced by the presence of growing dust particles," IEEE Trans. Plasma Sci., vol. 36, p. 1012, 2008.

[25] M. Mikikian, L. Couëdel, Y. Tessier, and L. Boufendi, "Carousel instability in a capacitively-coupled rf dusty plasma," IEEE Trans. Plasma Sci., vol. 39, p. 2748, 2011.

[26] H. Tawidian, M. Mikikian, L. Couëdel, and T. Lecas, "Plasma inhomogeneities near the electrodes of a capacitively-coupled radio-frequency discharge containing dust particles," Eur. Phys. J. Appl. Phys., vol. 56, p. 24018, 2011.

[27] A. P. Nefedov, G. E. Morfill, V. E. Fortov, H. M. Thomas, H. Rothermel, T. Hagl, A. Ivlev, M. Zuzic, B. A. Klumov, A. M. Lipaev, V. I. Molotkov, O. F. Petrov, Y. P. Gidzenko, S. K. Krikalev, W. Shepherd, A. I. Ivanov, M. Roth, H. Binnenbruck, J. Goree, and Y. P. Semenov, "Pke-nefedov: plasma crystal experiments on the international space station," New $J$. Phys., vol. 5, p. 33, 2003.

[28] J. Schulze, D. Luggenhölscher, and U. Czarnetzki, "Instabilities in capacitively coupled radio-frequency discharges," IEEE Trans. Plasma Sci., vol. 36, p. 1402, 2008.

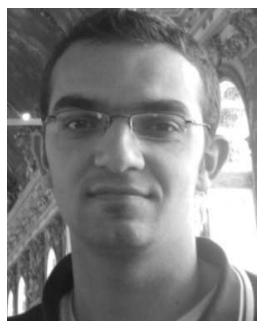

Hagop Tawidian was born in 1986 in Mdawar, Lebanon. He received the M.S. degree in microwave, electromagnetism and optoelectronic from the Université Paul Sabatier, Toulouse, France in 2010.

$\mathrm{He}$ is currently working toward the Ph.D degree at GREMI (CNRS/Université d'Orléans).

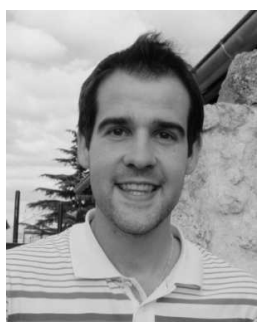

Thomas Lecas was born in 1986 in Orléans, France. $\mathrm{He}$ received the Associate degree in electronics in 2006.

Since 2010, he has been a CNRS assistant engineer in instrumentation with GREMI (CNRS/Université d'Orléans).

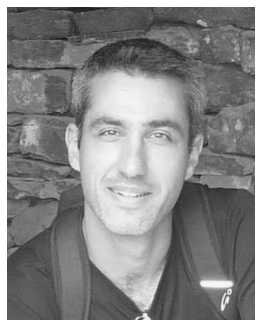

Maxime Mikikian was born in Marseille, France, in 1973. He received the Ph.D. degree in radiation and plasmas from the Université de Provence, Marseille, in 1999 and the Habilitation in physics from the Université d'Orléans in 2008.

Since 2004, he has been a CNRS researcher with GREMI (CNRS/Université d'Orléans).

His research interests include dusty plasmas and particularly instabilities, dust particle formation, dust cloud structure and dust charging. 